\title{
TUTORIZACIÓN INTERNACIONAL: abertura de horizontes para alcanzar nuevos mares
}

\author{
Janaína Soares Alves ${ }^{1}$ \\ Ana María Díaz Ferrero
}

\begin{abstract}
Resumen
En este artículo realizamos una propuesta para el desarrollo de la competencia comunicativa de los estudiantes de español como lengua extranjera del curso de Letras-Español de la Universidad de Brasilia (UnB) y de los estudiantes de portugués como lengua extranjera del grado de Traducción e Interpretación de la Universidad de Granada (UGR). Partiendo del hecho de que la inmersión lingüística es un marco acelerador de la competencia comunicativa de los estudiantes, presentamos en este artículo una propuesta didáctica para favorecer la interacción entre estudiantes brasileños y españoles. Se trata de colaborar con los programas de tutorización internacional existentes en estas dos universidades: el programa MENTOR en la UGR y el Programa de Tutores internacionais de la UnB. Se creará un grupo de trabajo colaborativo que establecerá una serie de actividades destinadas a facilitar la integración de los estudiantes brasileños en Granada y de los estudiantes españoles en Brasilia. De este modo se favorece la interacción $\mathrm{y}$, por consiguiente, mejora la competencia comunicativa de la lengua extranjera.
\end{abstract}

Palabras clave: internacionalización; intercambio universitario; inmersión lingüística.

\section{TUTORIA INTERNACIONAL: abertura de horizontes para alcançar novos mares}

\begin{abstract}
Resumo
Neste artigo realizamos uma proposta para o desenvolvimento da competência comunicativa dos estudantes de espanhol do curso de Letras-Espanhol da Universidade de Brasília (UnB) e dos estudantes de português como língua estrangeira da Faculdade de Tradução e Interpretação da Universidade de Granada (UGR). Partindo do fato de que a imersão linguística é um marco acelerador da competência comunicativa dos estudantes, apresentamos neste artigo uma proposta didática para favorecer a interação entre estudantes brasileiros e espanhóis. Trata-se de colaborar com os programas e tutoria internacional existentes nestas universidades: o programa MENTOR na UGR e o Programa de Tutores Internacionais da UnB. Será criado um grupo de trabalho colaborativo que estabelecerá uma série de atividades destinadas a facilitar a integração dos estudantes brasileiros em Granada e dos estudantes espanhóis em Brasília. Deste modo se favorece a interação e, em consequência, melhora a competência comunicativa da língua estrangeira.
\end{abstract}

Palavras-chave: internacionalização; intercâmbio universitário; imersão linguística.

\begin{abstract}
INTERNATIONAL TUTORING: opening horizons to reach new seas
Abstract

In this article we make a proposal for the development of the communicative competence of the students of Spanish as a foreign language of the Spanish-Language course of the University of Brasilia and of the students of Portuguese as the foreign language of the degree of Translation and Interpretation of the University of Granada. Starting from the fact that linguistic immersion is an accelerating framework of the communicative competence of students, we present in this article a didactic proposal to favor interaction between Brazilian and Spanish students. The aim is to collaborate with the existing international tutoring programs at these two universities: the MENTOR program at the UGR and the International Tutors Program at UnB. A collaborative work group will be

\footnotetext{
${ }^{1}$ Universidade de Brasília (UNB), Brasília - DF - Brasil. Professora adjunta. Doutora em Língua Espanhola. ORCID < https://orcid.org/0000-0001-9906-2647>. E-mail: janainasoares.unb@gmail.com

${ }^{2}$ Universidad de Granada (UGR), Granada - España. ORCID <https://orcid.org/0000-0002-0916-5552>. E-mail: anadiaz@ugr.es
} 
created that will establish a series of activities aimed at facilitating the integration of Brazilian students in Granada and Spanish students in Brasilia. In this way, interaction is favored and, therefore, the communicative competence of the foreign language improves.

Keywords: internationalization; university exchange; linguistic immersion

\section{Introducción}

Hasta hace menos de dos décadas el panorama para el aprendizaje de lenguas extranjeras (LE), más allá del material didáctico, se veía reducido a recursos limitados como casetes, cintas de vídeo y periódicos de difícil acceso y alto coste. La realización de estancias de inmersión era hace unos años una actividad que suponía en algunos casos una arriesgada "aventura" llevada a cabo con una logística complicada que incluía poco o escaso contacto previo con el país meta, largos viajes y dificultades de comunicación entre el país de origen y el de destino durante la estancia. Estas circunstancias dificultaban la realización de estancias de inmersión lingüística, por lo que en países como Brasil estaban restringidas casi exclusivamente a un grupo de estudiantes cuyo poder adquisitivo les permitía viajar al extranjero.

Este panorama está cambiando debido fundamentalmente a dos factores que han supuesto una revolución en la enseñanza y el aprendizaje de LE: el creciente uso de Internet y las políticas de internacionalización de las diferentes universidades. Como resultado de estos elementos transformadores observamos por un lado, que las nuevas tecnologías, el uso masivo de las redes sociales y los medios de comunicación digitales permiten el acceso a textos reales en distintas lenguas, realizar actividades de inmersión virtual en el proceso de aprendizaje de LE y agilizar el proceso de preparación de intercambios universitarios. Por otro lado, los diferentes programas de internacionalización del ámbito universitario permiten implementar proyectos de colaboración investigadora de profesores, y crear redes y grupos de intercambio para facilitar la movilidad de estudiantes.

Este es el caso del programa $\mathrm{MENTOR}^{3}$ ideado para poner en contacto a estudiantes de la Universidad de origen con estudiantes de la Universidad de destino para que actúen como mentores o tutores internacionales durante todo el proceso y faciliten la integración cultural, académica y lingüística. En este artículo exponemos las características de un proyecto de colaboración interuniversitario entre la Universidad de Brasilia (UnB) y la Universidad de Granada (UGR) para facilitar la integración durante los intercambios y favorecer la inmersión lingüística a través del programa.

\footnotetext{
${ }^{3}$ Disponible en: https://internacional.ugr.es/pages/perfiles/estudiantes/programa-mentor-de-la-ugr. Acceso en: 27 feb. 2019.
} 


\section{Fundamentos de la propuesta}

El gobierno brasileño ha apostado en las últimas décadas por la creación y desarrollo de programas de intercambio para expandir e internacionalizar la ciencia, la tecnología y la innovación. Así, a través del Ministério da Educação (MEC), el Ministério das Relações Exteriores (MRE) y el Ministério de Ciência e Tecnologia (MCT) se están adoptando estrategias y programas para la internacionalización de las instituciones de enseñanza superior (IES) en Brasil. Para obtener el máximo beneficio de estas políticas de expansión, tales instituciones deben definir sus socios colaboradores nacionales e internacionales y presentar propuestas específicas de colaboración. Como señala el informe sobre internacionalización de las universidades brasileñas elaborado por la Diretoria de Relações Internacionais (2017):

[As IES] deverão contar com infraestrutura para internacionalização, utilização de idiomas estrangeiros, escritório de relações internacionais, projetos para receber estudantes/pesquisadores estrangeiros, treinamento da sua equipe técnica, apropriação do conhecimento adquirido pelo bolsista após o retorno ao país, entre outras ações, que vão além da mobilidade ativa e passiva de docentes e discentes, construindo um ambiente internacional no dia a dia da Universidade. (BRASIL, 2017, p. 7)

Además de los convenios marco con la Universidad de Granada, diversas IES brasileñas tienen actualmente centenares de convenios específicos y de cotutela, lo que significa que cada año más estudiantes de Brasil se desplazan a Granada a través de programa de movilidad. Participar en uno de estos programas de intercambio, además de ser una experiencia personal y académica enriquecedora, supone obtener los beneficios derivados de un proceso de inmersión lingüística. Como afirma Knight (2015, p. 2, apud ALVES, 2017, p. 64), la internacionalización de la educación superior no debe ser un fin en sí mismo, sino un "meio para melhorar ou alcançar os objetivos acadêmicos da instituição ou as metas socioculturais, econômicas ou políticas do país ou região". Así, esta propuesta metodológica se inserta dentro de un programa de internacionalización cuyo objetivo es facilitar la integración de los estudiantes brasileños y/o españoles que participan en un programa de movilidad.

Muchas veces aprender una LE en Brasil representa reproducir formas y contenidos de un "otro" invisible y desvinculado de su contexto real: es como enseñar y aprender una lengua desde un vacío de cultura, de significados y de enriquecimiento recíproco, aspectos fundamentales para la adquisición y el aprendizaje de una lengua viva. Los intercambios universitarios funcionan como un elemento de enriquecimiento cultural, de ampliación de visiones y de fomento de la alteridad, aspectos estos que hacen que se perciba al "otro" como 
parte de un mundo multicultural y transfronterizo. Los intercambios permiten desarrollar lo que la UNESCO describe cómo los cuatro pilares del conocimiento humano, y a los responsables de la educación le corresponde propiciarlos: aprender a conocer, es decir, adquirir los instrumentos de la comprensión inter e intrapersonal, lo que lleva a la alteridad; aprender a hacer, para poder influir sobre el entorno; aprender a vivir en conjunto, para participar y cooperar con los demás en las diversas actividades; y, finalmente, aprender a ser: un proceso fundamental de la existencia que depende del desarrollo de los tres anteriores. Nuestra propuesta posibilita este conocimiento y, como reflexiona Delors (1996, p. 91), en la educación actual estas son cuatro vías del saber que se convergen en una sola, ya que hay entre ellas múltiples puntos de contacto, coincidencia y permutas.

En relación a los beneficios de participar en un proceso de inmersión lingüística está demostrado que es un marco acelerador de la competencia comunicativa de los estudiantes. Investigadores como Serrador (2016) han analizado los cambios lingüísticos observados en los estudiantes que participan en un programa de inmersión y concluye que las ventajas son múltiples y evidentes. Lograr enseñar y aprender LE en contextos reales es hoy una ventaja y poco a poco resulta fundamental para la contextualización de los diversos contenidos que forman la competencia comunicativa (HYMES, 1972). Según las premisas de este autor, el estudiante debe desarrollar la capacidad de crear, producir y descodificar mensajes, discursos apropiados, orales y escritos, que sean efectivos en la comunicación e interacción con su interlocutor. La competencia comunicativa supone, por lo tanto, el reconocimiento de la situación comunicativa y la adecuación de los registros y estilos comunicativos del interlocutor, lo que significa que el estudiante necesita desarrollar no solo el conocimiento sociocultural, contextual, pragmático, sino también el lingüístico o gramatical (formado por los componentes fonológico, sintáctico, semántico, léxico y morfológico), para expresar adecuadamente significados y negociarlos con su interlocutor. En definitiva, la existencia de un interlocutor es fundamental en el proceso de desarrollo de la competencia comunicativa en LE.

Otro factor importante en el proceso de enseñanza y aprendizaje de una lengua que se debe tener en cuenta es la motivación ya que está directamente relacionada tanto con los factores cognitivos e individuales como con los sociales (LORENZO, 2006). La motivación está vinculada a la memoria, por lo tanto, altos niveles de motivación hacen que el estudiante vincule conocimientos, haga asociaciones, emplee frases con creatividad y con sentidos diversos (WENDEN; RUBIN, 1987), lo que se logra también con la presencia del "otro" en el proceso de adquisición y aprendizaje de la LE. De esta manera, los programas de intercambio 
son de gran utilidad para estimular la motivación de los estudiantes dado que se integran en un ambiente real de la lengua meta y pueden realizar actividades de interacción de manera natural. La propuesta metodológica que presentamos en este artículo consiste en realizar un trabajo colaborativo interuniversitario que sirva de apoyo para la integración de los estudiantes que realizen una estancia, y al mismo tiempo ayude a desarrollar la competencia comunicativa en LE de los miembros integrantes en el grupo, es decir, español para los estudiantes brasileños y portugués para los estudiantes españoles.

\section{Desarrollo de la competencia comunicativa a través del programa MENTOR}

El programa MENTOR (buddy system, buddy programme o buddy scheme, en inglés) es un programa de apoyo para los estudiantes de intercambio existente en numerosas universidades. En la UGR este programa está coordinado desde el Vicerrectorado de Relaciones Internacionales y Cooperación al Desarrollo de la Universidad de Granada. En esta Universidad se inició como una experiencia piloto durante el curso 2011-12 y, debido a la gran acogida que tuvo entre los estudiantes, se activa cada año con resultados excelentes de participación. Este programa pone en contacto a estudiantes de la Universidad de Granada con estudiantes internacionales que van a realizar un intercambio académico en la UGR para que actúen como mentores y presten ayuda a los estudiantes extranjeros en tareas como asesoría en procesos administrativos, ayuda para buscar alojamiento u orientación para facilitar la integración en la comunidad universitaria.

La UnB también ofrece un programa similar de ayuda a los estudiantes de intercambio. Se llama Programa de tutores internacionais. En estos términos se anuncia el programa en la página Assessoria de Assuntos internacionais de la UnB:

\section{PROGRAMA DE TUTORES INTERNACIONAIS}

Tutor internacional é um estudante voluntário selecionado para te ajudar desde a sua chegada na UnB e, principalmente, durante a sua permanência, tentando sanar as dificuldades encontradas nos mais diferentes âmbitos. O principal objetivo é promover a integração acadêmica e social dos estudantes estrangeiros junto à comunidade local, através do companheirismo e da solidariedade. Se você quiser ter um "tutor", favor, entrar em contato com nossa equipe pelo e-mail intweb@unb.br. (Disponible en: http://www.int.unb.br/br/estude-na-unb/tutores-internacionais. Acceso el 27 feb. 2019) 
Nuestro proyecto consiste en crear un grupo de trabajo colaborativo entre los estudiantes de español como lengua extranjera de la licenciatura de Letras-Español de la UnB y los estudiantes de portugués como la lengua extranjera del grado de Traducción e Interpretación de la UGR. Este grupo de trabajo colaborará con el programa MENTOR de la UGR y con el programa de tutores internacionais de la UnB para establecer un protocolo de actuación destinado a asesorar para la realización de los intercambios, ayudar durante la estancia, facilitar la integración y proporcionar apoyo lingüístico.

\section{Contexto de aplicación}

La UnB, fundada en 1962, es una de las universidades brasileñas con mejor rendimiento académico de Brasil, aparece en el noveno lugar en el Ranking Universitário Folha (RUF) de Brasil de 2018, y en el decimonoveno lugar en QS World Rankings (2018) de universidades de América Latina. Desde la firma del convenio marco entre la UGR y la UnB en 2010, los resultados de internacionalización, enseñanza coordinada y proyectos de investigación no han dejado de aumentar.

Unos de estos proyectos es el exponemos en este trabajo llevado a cabo entre el curso de Letras de la UnB y el grado de Traducción e Interpretación de la UGR. En el curso de Letras de la UnB los estudiantes reciben una formación para obtener el título de profesores de lengua española de enseñanza secundaria. En su mayoría son de nacionalidad brasileña y estudian la lengua española durante los 4 años de la carrera con asignaturas que asocian el aprendizaje de la lengua y su aplicación didáctica para la práctica de la profesión docente. Al comenzar este curso de Letras los estudiantes no están obligados a tener conocimientos previos de lengua española, aunque cada vez hay más estudiantes que ingresan en la Universidad tras haber estudiado español en la enseñanza secundaria. En el curso de Letras de la UnB tienen que cursar 46 créditos de lengua española incluyendo las asignaturas de gramática y lenguas para fines específicos, 12 créditos de literatura y 2 asignaturas de prácticas docentes. Además, completan su formación con otras asignaturas de Lingüística, Psicología y Educación.

En el Grado en Traducción e Interpretación de la Facultad de Traducción e Interpretación (FTI) de la UGR los estudiantes reciben una formación como traductores generalistas e intérpretes de enlace en dos lenguas extranjeras. Una primera lengua, llamada lengua B y en la que es imprescindible que tengan un nivel de competencia lingüística B1, según el Marco Común Europeo de Referencia para las lenguas (MCER), y una segunda 
lengua, llamada lengua $\mathrm{C}$, entre las que se encuentra el portugués y para la que no es obligatorio tener conocimientos previos. Los estudiantes que escogen el portugués como lengua $\mathrm{C}$ tienen que realizar obligatoriamente 42 créditos de lengua portuguesa, 6 créditos de Cultura portuguesa y 18 créditos de asignaturas de traducción. Por otro lado, para completar su formación pueden elegir hasta 18 créditos más de asignaturas optativas relacionadas con la lengua portuguesa. La mayoría de los estudiantes en el Grado de Traducción e Interpretación de la UGR empiezan su formación lingüística en portugués el primer año de la carrera y una vez realizadas todas las asignaturas de lengua portuguesa alcanzan el nivel lingüístico necesario para poder desempeñar tareas de traducción e interpretación.

El uso de la LE en situaciones reales y con hablantes nativos es muy reducido tanto en el caso de los estudiantes brasileños como en el caso de los estudiantes españoles, por lo que la participación en programas de intercambio de movilidad es un complemento de gran utilidad para mejorar la competencia comunicativa en la LE. No obstante, conviene señalar que muchos estudiantes universitarios no consiguen participar en estos programas de intercambio internacional por presentar dificultades económicas para llevar a cabo un desplazamiento de alto coste. Por ello, esta propuesta didáctica supone una oportunidad para interactuar con estudiantes nativos en un contexto real y sin salir de la universidad de origen.

\section{Objetivos}

Como afirman Martins et al. en un artículo sobre cooperación internacional

[...] para desenvolver um bom trabalho de cooperação internacional, os estudiosos recomendam que as instituições sejam claras sobre seus objetivos, desenvolvam diretrizes e políticas, além de manter um sistema de investimento contínuo, tanto no que toca aos recursos financeiros como aos recursos humanos" (MARTINS et al, 2017, p. 63).

Por consiguiente, para implementar este proyecto se creará un grupo de trabajo colaborativo virtual que desempeñará diferentes actividades destinadas a conseguir una óptima integración de los estudiantes universitarios brasileños en Granada y de los estudiantes universitarios españoles en Brasília. Para conseguir este objetivo los estudiantes realizarán diferentes tareas individuales y colaborativas que conllevan el uso de la LE. Es decir, la cooperación de los estudiantes de la UGR en el programa MENTOR, y de los estudiantes de la UnB en el programa de tutores internacionais crea un escenario idóneo para llevar a cabo 
una metodología didáctica que implica tareas de documentación de la lengua y la cultura extranjera, así como la interacción oral y escrita con nativos de la LE.

Es por lo tanto un proyecto que contempla un doble objetivo: por un lado, conseguir la integración de los estudiantes internacionales a través de la colaboración de estudiantes mentores/tutores, y por otro, desarrollar la competencia comunicativa en la LE. Para ello proponemos una secuencia didáctica dividida en tres fases e integrada en el proceso de aprendizaje reglado de la formación universitaria de estos estudiantes.

\section{Secuencia didáctica}

Las asignaturas del grado de Traducción e Interpretación de la UGR en las que se incorporarán actividades para llevar a cabo esta propuesta son: Lengua C4 portugués, Lengua C5 portugués, Lengua C6 portugués, Cultura de la Lengua C (portugués), Traducción C1 (portugués), Traducción C2 (portugués) y Traducción C3 (portugués). En el caso de la UnB se incorporarán actividades en las siguientes asignaturas: Gramática Comparada; Teoria e Prática do Espanhol Oral e Escrito 1, 2 y 3, Gramática da Língua Espanhola y Usos Especializados do Espanhol. Los trabajos que deberán realizar los estudiantes se integran en tres fases de actuación:

Fase I. Elaboración de una guía informativa bilingüe

En cada universidad se creará un grupo de trabajo para elaborar un documento bilingüe que sirva de guía informativa para los estudiantes que participen en un programa de movilidad. En la UnB se creará una guía para la integración de los estudiantes universitarios españoles que realizan una estancia en Brasilia, y en la UGR se elaborará una guía informativa para los estudiantes brasileños que participan en un programa de movilidad en Granada. El objetivo de esta guía es informar de todo el proceso de movilidad y facilitar la integración de los estudiantes internacionales en las respectivas universidades de acogida. Para ser un buen anfitrión hay que conocer al visitante; por ello se establecerán grupos de trabajo entre los estudiantes de la UnB y la UGR para conocer las necesidades de los estudiantes de cada país y los posibles choques o problemas de integración que puedan surgir. Esta guía informativa deberá contener información relativa a los asuntos administrativos y académicos de la universidad de acogida, posibilidades de alojamiento, datos informativos sobre aspectos geográficos y socioculturales (gastronomía, clima, horarios, turismo, ocio...) y una pequeña guía con información lingüística. 
Para su elaboración se utilizarán todas las herramientas virtuales posibles como el chat, WhatsApp o messenger, además de las diversas formas de intercambio vía tándem, de propuestas de aprendizaje colaborativo distribuido en sus diversas formas de ejecución vía Internet. A través de un ordenador se pueden realizar tareas de documentación, intercambio de correos o debates por videoconferencia, entre otras (SOUZA, 2003; SILVA, 2008; SALOMÃO, SILVA, DANIEL, 2009). El uso de estos recursos en el aula es una actividad frecuente y necesaria para el proceso de enseñanza y aprendizaje de lenguas extranjeras. No obstante, las situaciones de comunicación forzadas y artificiales pueden provocar una actitud de rechazo o desmotivación en los estudiantes.

En este sentido, consideramos que esta propuesta didáctica para elaborar y publicar una guía informativa bilingüe será un estímulo para los estudiantes ya que estarán involucrados en situaciones de comunicación real para la consecución de un objetivo no meramente artificial y académico, sino real y profesional.

Por otra parte, durante esta primera fase se crearán también grupos de trabajo para atender las dudas que puedan plantear los estudiantes internacionales en la etapa de preparación de su intercambio. Para agilizar este proceso se elaborará un documento de con preguntas frecuentes (FAQ) para la UGR y otro para la UnB.

Fase II: Bienvenida e integración de los estudiantes

La segunda fase comienza con la llegada de los estudiantes de intercambio a la UnB y a la UGR. Se creará una agenda de actividades en cada una de las universidades de acogida para facilitar la integración de los estudiantes extranjeros: jornada de bienvenida, encuentros y talleres de inmersión lingüística e integración cultural. Dado que los estudiantes que se desplazan a Granada y a Brasilia provienen de distintas áreas y muchos de ellos no han realizado un curso específico de LE, estos talleres servirán de ayuda a los estudiantes extranjeros para mejorar sus conocimientos de la LE. Los estudiantes del curso de Letras de la UnB y los estudiantes del curso de Traducción e Interpretación de la UGR actuarán como asesores lingüísticos en estos talleres.

En esta fase los estudiantes mantienen un contacto directo y presencial con interlocutores de la LE. Como afirma Jauregi (2012, p. 6) la interacción aúna las dos condiciones necesarias para fomentar la adquisición: la recepción de input y la producción de output comprensibles. De este modo, la exposición del estudiante a un input variado y rico en la lengua meta, así como la interacción con interlocutores nativos les permite ampliar su competencia léxica, sociocultural y pragmática necesarias para realizar un uso adecuado de la 
lengua. Es decir, se crean situaciones de inmersión en las que el estudiante se convierte en un observador y actor directo, y adquiere la información necesaria para identificar los rasgos diferenciales diafásicos y diatópicos de los interlocutores, así como el léxico y las estructuras lingüísticas adecuadas para cada situación comunicativa.

Para obtener un máximo aprovechamiento de estas interacciones entre estudiantes brasileños y españoles se realizarán actividades para despertar la conciencia lingüística de los estudiantes implicados, esto es, se fomentará la realización de actividades para realizar un aprendizaje consciente, reflexiones sobre la lengua, sus reglas y sus componentes.

Fase III: Elaboración de un informe

Al finalizar cada año académico, los estudiantes elaborarán un informe que servirá de base para mejorar las prestaciones del programa MENTOR de la UGR y del programa de tutores internacionais de la UnB.

Los estudiantes extranjeros que hayan participado en un programa de movilidad responderán a un cuestionario para valorar su intercambio. Con los resultados obtenidos y las reflexiones personales de los estudiantes mentores/tutores se realizará un informe valorativo final que ofrecerá propuestas de mejora para los próximos años.

\section{Consideraciones finales}

A partir de las aportaciones teóricas consideradas en este artículo y de las posibilidades que la organización de la sociedad mundial ofrece, se hace casi que necesaria la inmersión lingüística como acelerador del desarrollo de la competencia comunicativa en LE del estudiante. Este es el objetivo principal de la propuesta didáctica que hemos presentado en este artículo, lo que se logrará a través de la participación en un programa interuniversitario que conlleva la realización de tareas de inmersión lingüística.

Los nuevos retos del conocimiento actualmente demuestran que estrechar los vínculos y acercarse al otro es un factor importante para el desarrollo del ser humano en sus dimensiones intra e interpersonal y todo eso se observa en las actividades que conforman nuestra propuesta de intercambio.

De esta manera, no solo se fomenta el desarrollo de las diversas competencias que forman la competencia comunicativa de los estudiantes (competencias lingüísticas, sociolingüísticas y pragmáticas), sino también se les ofrece una oportunidad de agrandar su visión hacia un otro que no es invisible, sino parte de su proceso individual de crecimiento y de desarrollo de habilidades variadas. 


\section{Referencias}

ALVES, G. K. Avaliação da internacionalização da educação superior: proposição de indicadores e mapeamento de procesos. Navus. Florianópolis/SC, v.7, n.4, p. 63-76, out./dez., 2017. Disponible en: http://dx.doi.org/10.22279/navus.2017.v7n4.p63-76.549. Acceso en: 27 feb. 2019.

BRASIL, Ministério da Educação. Coordenação de Aperfeiçoamento de Pessoal de Nível Superior. A internacionalização na Universidade Brasileira: resultados do questionário aplicado pela Capes. Brasília: Diretoria de Relações Internacionais. 2017.

DELORS, J. Los cuatro pilares de la educación. In: DELORS, J. (org.) La educación encierra un tesoro. Informe a la UNESCO de la Comisión internacional sobre la educación para el siglo XXI, Madrid: Santillana/UNESCO, 1996, p. 91-103.

HYMES, D. H. Acerca de la competencia comunicativa. (1972) In: LLOBERA et al. Competencia comunicativa. Documentos básicos en la enseñanza de lenguas extranjeras. Madrid: Edelsa, 1995.

JAUREGI, K. La interacción, elemento clave en el proceso de aprendizaje de E/LE. In: XXIII Congreso Internacional de la ASELE Plurilingüismo y enseñanza de ELE en contextos multiculturales. Girona: Asociación para la Enseñanza del Español como Lengua Extranjera, 2012, p. 5-16.

KRASHEN, S.D. Principles and practice in second language acquisition. Oxford: Pergamon, 1972.

KRASHEN, S.D.; TERRELL, T. The Natural Approach: Language Acquisition in the Classroom. Oxford: Pergamon, 1984.

LORENZO, F. Motivación y segundas lenguas. Madrid: Arcolibros, 2006.

MARCO COMÚN EUROPEO DE REFERENCIA PARA LAS LENGUAS: [en línea]. Disponible en: https://cvc.cervantes.es/ensenanza/biblioteca_ele/marco/cvc_mer.pdf. Acceso en: 27 feb. 2019.

MARTINS, V.; MONTAGUE, A.; SILVA, P. B. da Cooperação internacional para mobilidade estudantil: o caso da Umesp e da Zuyd. Revista de Educação do Cogeime, Ano 26, n. 50, janeiro-junho, 2017, p. 59-79. Disponible en: https://www.redemetodista.edu.br/revistas/revistascogeime/index.php/COGEIME/article/view/705. Acceso en: 27 feb. 2019.

PERICO, F. G.; GONCALVES, R. B. Intercâmbio acadêmico: as dificuldades de adaptação e de readaptação. Educ. Pesqui., São Paulo, v. 44, e182699, 2018. Disponible en http://www.scielo.br/scielo.php?script=sci_arttext\&pid=S1517-

$97022018000100483 \& \operatorname{lng}=$ pt\&nrm=iso. Acceso en: 27 feb. 2019.

SALOMÃO, A. C. B.; SILVA, A. C. ; DANIEL, F. G. A aprendizagem colaborativa intandem: Um olhar sobre seus princípios. In: TELLES, J. A. (org.). Teletandem: um contexto 
virtual, autônomo e colaborativo para a aprendizagem de línguas no século XXI. 1ed., Campinas: Pontes, 2009, p. 75-92.

SERRADOR, A. R. Los programas de intercambio como herramienta de aprendizaje en contextos de enseñanza plurilingüe. In: DÍEZ, M. A. et ali (eds.) Aprendizajes pluriligües y literarios. Nuevos enfoques didácticos. Alicante: Publicaciones de la Universitat d'Alacant, 2016 , p. 208-215, Disponible en: https://rua.ua.es/dspace/bitstream/10045/64739/1/Aprendizajes-plurilingues-yliterarios_23.pdf. Acceso en: 27 feb. 2019.

SILVA, A. C. O desenvolvimento intra-interlinguístico in tandem a distância (Português e Espanhol). Dissertação (Mestrado em Estudos Linguísticos) - Instituto de Biociências, Letras e Ciências Exatas da Universidade Estadual Paulista, UNESP, São José do Rio Preto, 2008, $358 \mathrm{p}$.

SOUZA, R. A. Telecolaboração e divergência em uma experiência de aprendizagem de português e inglês como línguas estrangeiras. In: Revista Brasileira de Lingüística Aplicada, Belo Horizonte, v. 3, n. 2, 2003, p. 73-96.

STALLIVIERI, L. Internacionalização e intercâmbio: dimensões e perspectivas, Curitiba: Appris, 2017.

UNIVERSIDADE DE BRASÍLIA, Programa de tutores internacionais. In: Assessoria de Assuntos Internacionais. Disponible en: http://www.int.unb.br/br/estude-na-unb/tutoresinternacionais. Acceso en: 27 feb. 2019.

WENDEN, A.; RUBIN, J. (orgs). Learner Strategies in Language Learning. New Jersey: Prentice-Hall International. 1987. 